\title{
Contando Histórias com Realidade Aumentada
}

\author{
Livro Interativo para Crianças com Necessidades Especiais em Lábrea - AM
}

\author{
Francisca Kauana da Silva \\ Instituto Federal do Amazonas - \\ Campus Lábrea \\ Lábrea - Amazonas - Brasil \\ franciscakauanaalmeida.15@gmail.com
}

\author{
Mizael Nascimento Rodrigues \\ Instituto Federal do Amazonas - \\ Campus Lábrea \\ Lábrea - Amazonas -Brasil \\ mizaeldesigner13@gmail.com
}

\author{
Fabiann Matthaus Barbosa \\ Instituto Federal do Amazonas - \\ Campus Lábrea \\ Lábrea - Amazonas- Brasil \\ fabiann_matthaus@hotmail.com
}

\begin{abstract}
It is observed that the constant evolution of technology is taking education to new directions, emphasizing the use of new tools and providing effective improvements in the teaching / learning process. Augmented Reality (AR) technology can be a great tool in education and can also target children with special needs. AR applications in education can benefit all who have more or less needs and can become a useful and innovative tool in education. Thus, this paper aims to present the development of a system in AR through an interactive children's textbook telling the story of the municipality of Labrea, in the interior of Amazonas, exploring the difficulties of students with autism and cerebral palsy, showing the student interaction with abstract knowledge completely and facilitating the way to the understanding of theories and concepts.
\end{abstract}

\section{KEYWORDS}

Educação Inclusiva, Informática na Educação, Realidade Aumentada, Inclusão Digital

\section{CONTEXTUALIZAÇÃo}

Na sociedade contemporânea, a tecnologia é explorada em diversos ambientes e vem sendo empregada em distintos campos da ciência. Segundo Canto e Almeida [1], os avanços tecnológicos sempre trazem transformações a sociedade e alteram hábitos, formas de pensar, meios de se comunicar e nosso estilo de vida como um todo. Essas evoluções vêm se refletindo na educação e essas transformações tecnológicas aceleradas demandam uma reformulação nas práticas pedagógicas, que precisam incorporar esses avanços tecnológicos [2]. A utilização de novas tecnologias no espaço escolar pode proporcionar uma melhoria no processo de busca de informações e possibilitar uma comunicação mais ágil entre alunos, professores e instituições [3].

Diante do exposto, não há como dissociar o mundo da tecnologia e a educação, visto que houve um alargamento em relação ao uso de dispositivos tecnológicos na vida das pessoas e esses dispositivos chegaram às escolas. Entretanto, é possível observar que nem todas as instituições de ensino têm acesso as tecnologias. Com a falta das ferramentas tecnológicas, o professor muitas vezes as aulas em algo rígido, maçante e sem relação com o mundo real. A realidade é ainda mais difícil quando se trata de alunos que, por vários motivos, apresentam dificuldades de aprendizagem, o que pode decorrer de algum tipo de deficiência.

O artigo $3^{\circ}$ - Decreto $n^{\circ}$ 914/93 define deficiência como " $[$...] toda perda ou anormalidade de uma estrutura ou função psicológica, fisiológica ou anatômica que gere incapacidade para o desempenho de atividade, dentro do padrão considerado normal para o ser humano" [4]. Oliveira [5] evidencia que as tecnologias se tornaram indispensáveis para a qualidade e eficácia no processo de construção dos conhecimentos, visto que tem um papel essencial para todos os alunos, sendo, portanto, uma possível ferramenta de relevância no processo de aprendizado para alunos com necessidades especiais, pois favorecem uma maior motivação na aprendizagem e promovem a inclusão destes indivíduos na sociedade [5].

Por meio dos avanços tecnológicos ocorridos ao longo dos anos foi desenvolvida a Realidade Aumentada (RA), que surge com o propósito de facilitar a aprendizagem através da inserção de informações, projetando dados virtuais em ambientes do mundo real com apoio de dispositivos que aprofundam o entendimento dos indivíduos sobre determinado conteúdo, conforme Anami [6]. A RA envolve tecnologias revolucionárias para o desenvolvimento de aplicações, navegação em espaços tridimensionais, imersão no contexto da aplicação e interação em tempo real.

Segundo Cardoso [7], os recursos em RA podem propiciar um incremento na aprendizagem como: apoiar e motivar o aprendizado, fornecer cenários virtuais semelhantes aos reais acrescidos de informações complementares e ainda permitir a simulação e a análise de experiências não vivenciadas.

Para o ensino de crianças com necessidades especiais pode ter como vantagens: despertar o interesse e a motivação pela descoberta do conhecimento, oferecer a capacidade de se colocar dentro do ambiente virtual apresentado e desenvolver as capacidades intelectuais tais como a criatividade e a eficácia.

\section{PROBLEMÁTICA E JUSTIFICATIVA}

A cidade de Lábrea está localizada no sul do Estado do Amazonas e é banhada pelo rio Purus que exerce um papel fundamental na vida labrense, servindo de base hídrica que configura a vida 
município. Contudo, a mesma bacia hidrográfica que banha e alimenta a cidade também traz problemas de ordem logística que constituem grande desafio para a população. Entre os meses de dezembro e junho, habitualmente a cidade se vê isolada devido às cheias cíclicas do Purus, o transporte rodoviário torna-se insustentável a medida que as rodovias literalmente tornamse vastos lamaçais, sendo assim o transporte aéreo cumpre papel importante, mas seu acesso é limitado às camadas média e alta da população.

Por esse e outros aspectos o município possui baixo avanço tecnológico, carecendo de instrumentos e subsídios que possam levar o desenvolvimento da tecnologia na cidade. Essa falta de estrutura tecnológica é também evidente nas escolas municipais na cidade o que acaba muitas vezes refletindo no ensino e no desenvolvimento do aluno.

A Secretaria Municipal de Educação e Cultura (SEMEC) em Lábrea (figura 1) a fim de atender a população de baixa renda criou um projeto capaz de assistir famílias que possuem crianças e adolescentes com necessidades especiais com a finalidade de realizar um atendimento especializado inserindo essa parte da população ao convívio da sociedade. Para isso foi formada uma equipe multidisciplinar composta por: terapeuta ocupacional, fonoaudióloga, assistente social e psicóloga.

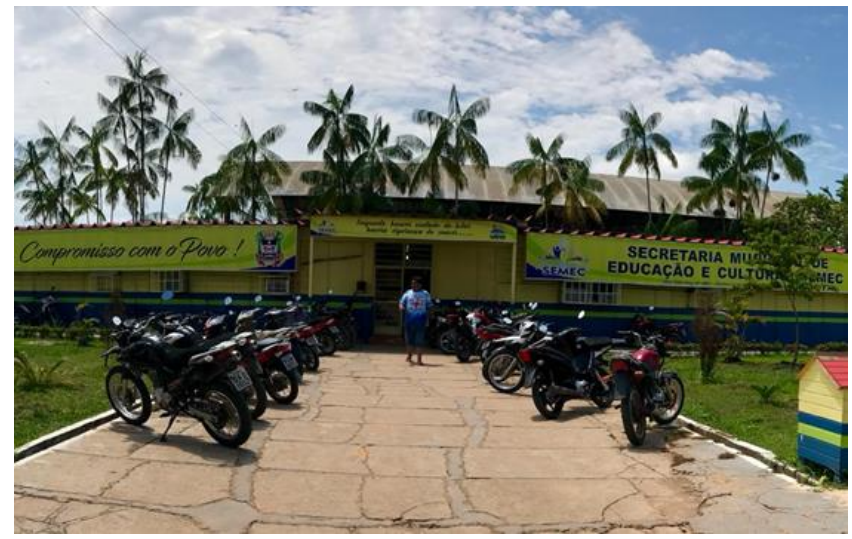

Figura 1. SEMEC no município de Lábrea - AM

Atualmente são atendidas 18 crianças com faixa etária entre 4 a

16 anos todas com necessidades especiais, tais como: autismo, dificuldades na fala e linguagem, deficiência auditiva/surdez, paralisia cerebral e com Transtorno do Déficit de Atenção com Hiperatividade - TDAH. As atividades com alunos são limitadas aos mesmos jogos educativos disponibilizados pela prefeitura como: ábaco, livros para colorir, jogos de memória, jogos de sombra, quebra-cabeça, caça-palavras e formação de palavras.

Os atendimentos ocorrem de forma individual com cada criança uma vez por semana no período de uma hora, pois assim, segundo a assistência social, o aluno tem um atendimento de todos os profissionais de maneira que possam explorar as dificuldades de cada indivíduo e desenvolver maneiras para trabalhar de
Sendo assim, propõe-se um livro didático interativo contando a história do local onde as crianças PNE's vivem de forma lúdica utilizando RA para dispositivos móveis. Portanto, procurase responder as seguintes questões: Como a RA pode ser um meio para estimular o aprendizado nas escolas? De que forma a RA pode ser usada para potencializar o desenvolvimento e habilidades dos alunos portadores de necessidades especiais?

\section{SOLUÇÃO PROPOSTA}

Partindo desse pressuposto, emergiu a ideia de um projeto capaz de proporcionar um ambiente diferente para esses estudantes que são amparadas junto a SEMEC em Lábrea, desenvolvendo um livro didático interativo contando a história do município de Lábrea de forma lúdica utilizando RA para dispositivos móveis promovendo a inclusão social por meio das histórias contadas e uma evolução no ensino, visto que as ferramentas tecnológicas motivam crianças e adolescentes ao aprendizado em virtude do seu poder de interesse e imersão proporcionadas.

A pesquisa foi aplicada e explorada com 4 (quatro) estudantes autistas e 6 (seis) com paralisia cerebral, buscando investigar e analisar o impacto que a tecnologia de realidade aumentada pode causar no processo de aprendizagem e inclusão digital dessas crianças. Portanto, procurou-se responder as seguintes questões: Como a RA pode ser um meio para estimular o aprendizado nas escolas? De que forma a RA pode ser usada para potencializar o desenvolvimento e habilidades dos alunos com necessidades especiais?

\section{OBJETIVOS}

\subsection{Objetivo Geral}

Apresentar o desenvolvimento de um livro didático interativo contando a história do município de Lábrea para alunos do ensino fundamental com necessidades especiais, utilizando a tecnologia de realidade aumentada para dispositivos móveis.

\subsection{Objetivos Específicos}

- Analisar o impacto que a tecnologia em RA pode proporcionar no comportamento e desenvolvimento dos alunos autistas e com paralisia cerebral.

- Potencializar o desenvolvimento de habilidades dos alunos com necessidades especiais tais como a criatividade e a eficácia fornecendo cenários virtuais semelhantes aos reais acrescidos de informações complementares.

- Proporcionar aos educadores da SEMEC em Lábrea uma ferramenta capaz de integrar as crianças com necessidades especiais na sociedade atendendo às diferenças individuais de cada um.

\section{DESENVOLVIMENTO}

A pesquisa quanto a natureza do método, foi fundamentada na pesquisa aplicada, visto que apresentou um produto que pode 
proporcionar um melhor rendimento na aprendizagem em sala de aula. Quanto a abordagem, a pesquisa qualitativa teve como intuito de avaliar o comportamento de utilização da tecnologia, analisando as atitudes a partir da utilidade percebida e da facilidade de uso percebida, baseado no modelo de aceitação de tecnologia (TAM).

Para o autor Davis [8], as pessoas tendem a usar ou não uma tecnologia com o objetivo de melhorar seu desempenho em alguma atividade - utilidade percebida (PU), porém, mesmo que essa pessoa entenda que uma determinada tecnologia é útil, sua utilização poderá ser prejudicada se o uso for muito complicado, de modo que o esforço não compense o uso - facilidade de uso percebida (PEOU).

A avaliação qualitativa analisou o estudante a partir de dois métodos: observação no comportamento do aluno e entrevistas com os educadores. Lüdke e André [9] complementam que a vantagem da entrevista sobre outras técnicas é que ela permite a captação imediata e corrente da informação desejada, praticamente com qualquer tipo de informante e sobre os mais variados tópicos.

\subsection{Materiais e Ferramentas}

Para o desenvolvimento do livro interativo em RA foi necessário alguns softwares e recursos computacionais. O primeiro foi o Blender que é uma aplicação integrada que permite a criação de uma diversificada gama de conteúdos 2D e 3D oferecendo uma série de recursos que permitem elaborar complexas modelagens e avançadas animações [10]. Essa ferramenta foi fundamental no desenvolvimento dos objetos apresentados no livro, pois deu vida ao personagem do livro a partir de texturas e edições.

Outro software que foi utilizado no desenvolvimento do trabalho foi o Unity3D (figura 2) que se destaca por ter possibilidade de ampliação tridimensional e integração com ferramentas de Realidade Aumentada [10]. O desenvolvimento de aplicações com RA no Unity3D pode ser realizado com a utilização da ferramenta Vuforia, possuindo rápida detecção dos locais de objetos, amplo reconhecimento de marcadores, bem como a qualidade de renderização [11].

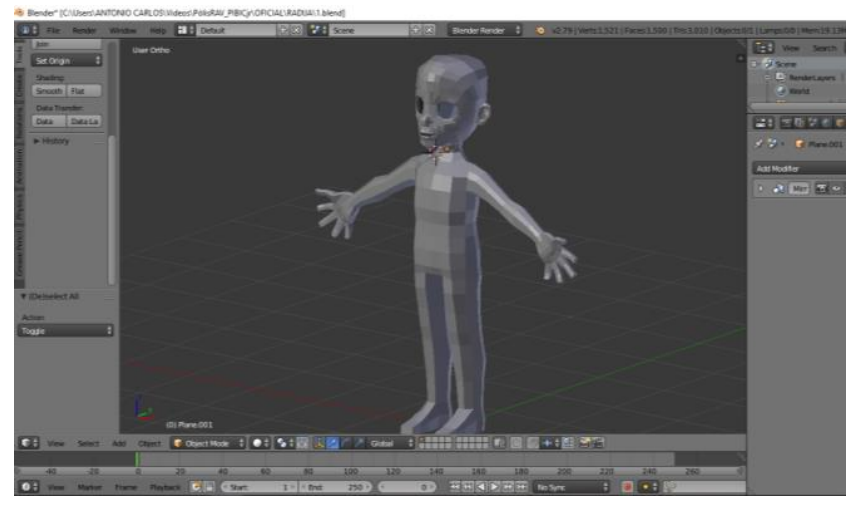

Figura 2. Capa do livro di dático interativo

De acordo com Leitão [9], o Vuforia possui uma documentação completa, além de reconhecer qualquer tipo de imagem, somente marcadores $2 \mathrm{D}$ e também é ágil para o reconhecimento de imagens/marcadores.

\section{PROCEDIMENTOS METODOLÓGICOS}

A pesquisa está dividida em duas fases distintas: implementação do livro e a aplicação no ambiente escolar. Sendo que a segunda fase complementa a primeira e que cada uma foi subdividida em pequenas etapas para o melhor desenvolvimento da pesquisa.

\subsection{Implementação do livro}

O ponto de partida foi a realização de um levantamento bibliográfico a fim de poder identificar as principais funcionalidades da RA, conhecer seus ambientes virtuais e estudar as plataformas de desenvolvimento para produção do livro.

Nessa etapa, também foi realizada uma pesquisa em acervos bibliográficos pela cidade que relatavam a história de Lábrea, assim como foram produzidos documentários em forma de entrevistas com pesquisadores e historiadores locais, como mostra a figura 3.

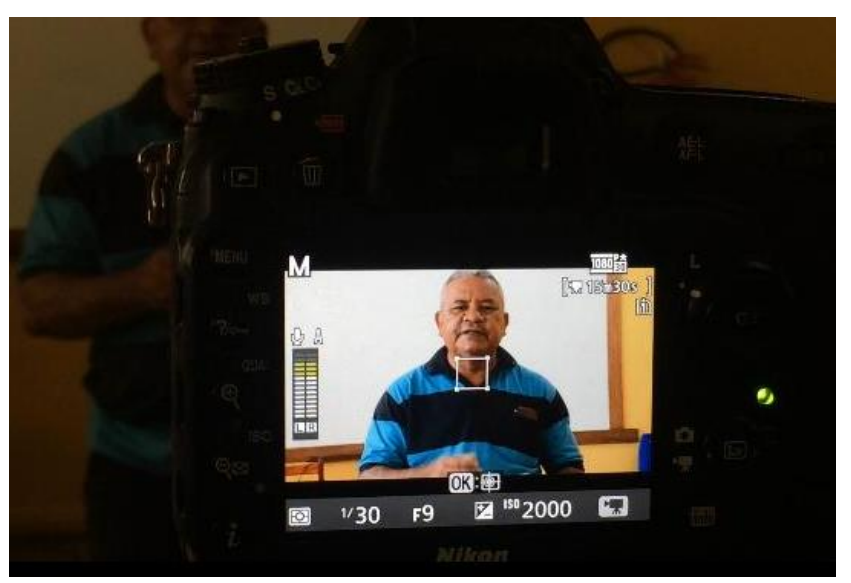

Figura 3. Entrevista com pesquisador sobre a história de Lábrea

Sendo assim, foram definidos todos os aspectos da estrutura do livro (figura 4), os conteúdos abordados e juntamente com a equipe multidisciplinar que atende as crianças foram acentuadas as melhores maneiras de contar as histórias utilizando objetos 3D e RA, com foco nas dificuldades dos alunos com autismo e paralisia cerebral.

Em continuidade foi realizada uma visita na SEMEC, com a finalidade de realizar uma reunião com os responsáveis dos alunos pedindo autorização para que os estudantes pudessem participar da pesquisa sem representar nenhum risco e mantendo o sigilo das informações de cada um.

Ao final da primeira fase do projeto foi realizada uma segunda reunião, agora com a equipe multidisciplinar, para apresentação do livro de forma que os mesmos pudessem expor suas considerações e sugestões para melhoria da ferramenta. Após a versão final do livro foram impressos exemplares para início das aplicações em sala de aula. 


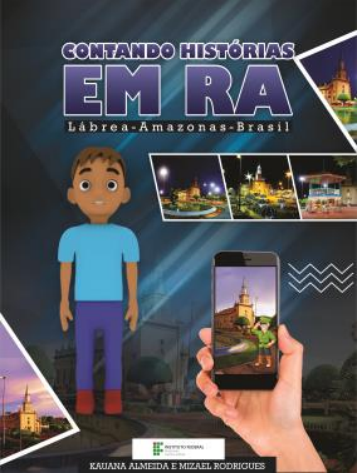

Figura 4. Capa do livro didático interativo

\subsection{Aplicação no ambiente escolar}

Para segunda fase da pesquisa, foi realizada a aplicação do livro com os alunos onde em cada encontro foi abordado uma história diferente e ao final de cada aula era aplicada uma atividade de fixação e revisão do assunto visto. Para que a criança não se sentisse incomodada ou constrangida com a presença dos pesquisadores, as histórias foram contadas pela própria equipe multidisciplinar.

Sendo assim, foram realizadas 11 (onze) aplicações com o livro e após o experimento foi realizada uma roda de conversa (figura 5) com os educadores que contaram as histórias para os alunos, com o objetivo coletar dados por meio de entrevistas sobre a compreensão de cada profissional no desenvolvimento do aluno, analisando assim os pontos positivos e negativos dos recursos tecnológicos explorados em sala de aula.

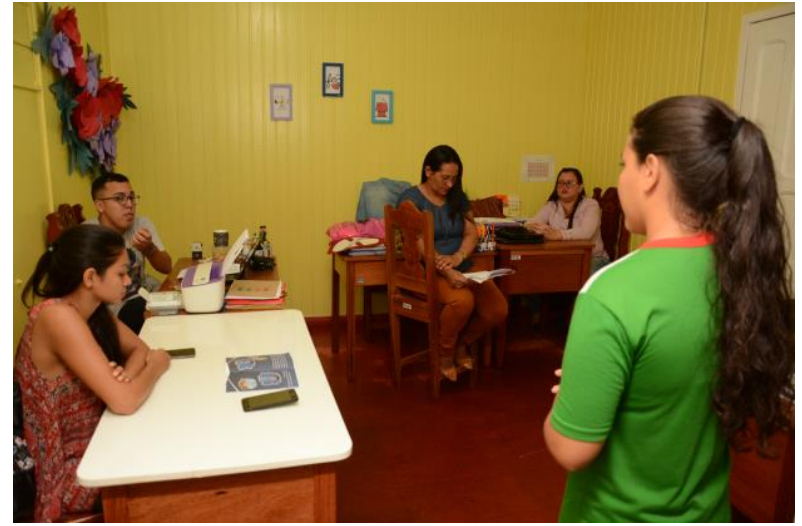

Figura 5. Roda de Conversa com a equipe multidisciplinar

\section{RESULTADOS E CONSIDERAÇÕES FINAIS}

Com o experimento realizado, através das rodas de conversa, os envolvidos na aplicação puderam estabelecer suas considerações sobre uso da RA em sala de aula, onde foram coletados os dados qualitativos seguindo o modelo TAM e com base nas perguntas abertas e fechadas em relação à utilidade da RA (figura 5) no aprendizado do aluno e o potencial da tecnologia para o desenvolvimento das habilidades dos estudantes com necessidades especiais. Além das entrevistas realizadas foram utilizados como métodos de coleta de dados, a observação através de registros realizados em cada atividade com o aluno.

\begin{tabular}{|c|c|}
\hline \multicolumn{2}{|c|}{ FACILIDADE DE USO } \\
$\mathbf{1}^{\mathbf{a}}$ & Foi fácil manipular a ferramenta na preparação e aplicação das aulas. \\
$\mathbf{2}^{\mathbf{a}}$ & Os alunos tiveram facilidade no uso do livro em sala de aula. \\
$\mathbf{3}^{\mathbf{a}}$ & O material para se utilizar em sala de aula é fácil acesso para escola. \\
$\mathbf{4}^{\mathbf{a}}$ & Os alunos tinham facilidade em compreender os conteúdos. \\
$\mathbf{1}^{\mathbf{a}}$ & Utilizaria essa ferramenta novamente em outros conteúdos? \\
$\mathbf{2}^{\mathbf{a}}$ & Com a utilização do livro o interesse dos estudantes aumentou nas aulas? \\
$\mathbf{3}^{\mathbf{a}}$ & $\begin{array}{l}\text { A utilidade do livro interativo facilitou na compreensão de utilizar novas } \\
\text { tecnologias para auxiliar no ensino? }\end{array}$ \\
\hline
\end{tabular}

\section{Figura 5. Questionário de pós-aplicação}

Os dados qualitativos coletados comprovam a facilidade no uso da ferramenta, além de demonstrar ser um recurso complementar para aprender e ensinar de forma lúdica e prazerosa contribuindo entre a teoria e a prática, entretanto os mesmos dados evidenciam que o uso dessa tecnologia está distante da realidade das escolas no município de Lábrea e que requer um conhecimento prévio para produção e criação dos ambientes em RA.

Portanto, pôde-se concluir que aplicação do livro em RA trouxe um maior interesse e motivação dos alunos e a partir das histórias contadas foi possível incluir esse público ao mundo digital e apresentar aos educadores o mundo de possiblidades que a tecnologia de realidade aumentada pode trazer, tornando o aprendizado mais atraente e instigante, viabilizando a adaptação de vários conceitos, favorecendo a motivação e assim desenvolvendo a imaginação do estudante.

\section{REFERENNCIAS}

11 Canto, T. S. do; Almeida R.D. (2014) M apas feitos por não cartógrafos e a prática cartográfica no ciberespaço. In: Novos rumos da cartografia escolar:currículo, linguagem e tecnologia/ Organização: Rosângela Doin de Almeida-São Paulo: Contexto.

[2] Carvalho, V. M. S. G. (2012) Sensoriamento Remoto no ensino básico da Geografia: definindo novas estratégias. Rio de Janeiro: APED.

[3] Freitas, R. V.; Lima, M. S. S. (2010). As novas tecnologias na educação: desafios atuais para a prática docente. $V$ Encontro de Pesquisa em Educação deAlagoas (V EPEAL). In: A nais... Alagoas. Disponível em: A cesso em: 10

[4] Maia, H. (2011). Org. Necessidades Educacionais Especiais. Rio de janeiro: Wak Editora.

[5] Oliveira, G. A. (2015). As TIC na motivação dos alunos com currículo específico individual. Dissertação (Mestrado em Ciências da Educação)Universidade Fernando Pessoa, Porto.

[6] A nami, B. (2013). Boas práticas de realidade aumentada aplicada à educação. Trabalho de Conclusão de Curso (Graduação). Bacharelado em Ciências daComputação - Universidade Estadual de Londrina. Londrina - PR. Disponívelem: A cesso em: 10 jun. 2019.

[7] Cardoso, R. et al. (2014). Uso da realidade aumentada em auxílio à educação. Computer on the Beach. Universidade Ceuma. São Luís - MA. p. 330-339. Disponível em: <file://C:/Users/aline/Downloads/5337-14326-1 SM \%20(1).pdf>. Disponível em: A cesso em: 12 jun. 2019.

[8] Davis. F. (1989) Perceived usefulness, perceived ease of use, and user acceptance of information technology. MIS Quarterly, p. 319-340.

[9] Ludke, M.; André, M. E. D. A. (1986) Pesquisa em educação: abordagens qualitativas. São Paulo: EPU.

[10] Blender. Disponível em http://wiki.blender.org/. Disponível em: A cesso em: 10 jun. 2019.

[11] Leitão, R. M. V. (2013). Aprendizagem baseada em jogos: realidade aumentada no ensino de sólidos geométricos. Universidade A berta, Programa dePós-Graduação em Expressão Gráfica e Audiovisual. Dissertação de Mestrado. 